\title{
Therapeutic potential of curcumin in medicinal chemistry
}

\author{
Daniela Koštálováa , Lýdia Bezákováb, Lucia Račkovác, \\ Silvia Mošovskáa ${ }^{a}$ Ernest Šturdík ${ }^{a}$ \\ ${ }^{a}$ Department of Nutrition and Food Assessment, Faculty of Chemical and Food Technology, \\ Radlinského 9, 81237 Bratislava \\ ${ }^{b}$ Department of Cell and Molecular Biology of Drugs, Faculty of Pharmacy, Comenius University, \\ Odbojárov 10, 83232 Bratislava \\ 'Institute of Experimental Pharmacology and Toxicology, Slovak Academy of Sciences, \\ Dúbrauská cesta 9, 84104 Bratislava \\ silvia.mosovska@gmail.com
}

\begin{abstract}
Extensive research over the past half century has shown that curcumin (diferuloylmethane), a polyphenolic compound of turmeric (Curcuma longa L.), can modulate multiple cell signaling pathways. Extensive clinical trials have addressed the pharmacokinetics, safety, and efficacy of this nutraceutical against numerous diseases in human. Curcumin, known for thousand years as a subject of Ayurvedic medicine, has undergone in recent times remarkable transformation into a drug candidate with prospective multipotent therapeutic application. Characterized by high chemical reactivity, resulting from an extended conjugated double bond system prone to nucleophilic attack, curcumin has been shown to interact with a plethora of molecular targets, in numerous experimental observations. In clinical trials, has been used either alone or in combination with other agent. However, its clinical advance has been limited by its short biological half-life, fast metabolism and poor systemic bioavailability after oral administration. To mitigate the above limitations, recently various formulation of curcumin, including nanoparticles, micelles, liposomes, phytosomes delivery system has been examined. The present review has been devoted towards better understanding of the phytonutraceutic properties of curcumin and turmeric based on their disease specific indications and enhancing their prophylactic and therapeutic nutraceutical qualities. The article deals with the biological activity, mode of action, toxicity and forthcoming application of these leads.
\end{abstract}

Keywords: curcumin, turmeric, absorption, bioavailability, metabolism, nanocurcumin, medicine

\section{Introduction}

Nutraceutical is a food or food product that reportedly provides health and medicinal benefit including the prevention and treatment of diseases. Diet has a major role in modulating the risk of development of several diseases. Edible plants, amongst dietary compounds, are important in that they contain phytochemicals which can control biochemical and physiological processes at cellular level of human organism. Among nutraceuticals, preparation of the turmeric (Curcuma longa L., family Zingiberaceae) rhizome have been used medicinally and as spices for millennia in several Asian countries and traditional Chinese medicine to treat bacterial infection, inflammation, burns, and digestive disorders. Some of the medicinal properties are attributed to curcumin (diferuloylmethane), a major polyphenolic compound of the rhizome (5-10\% of dry weight). Biomedical investigations of curcumin and curcuminoids have provided evidence of a wide range of molecular and cellular activities, most related to antiinflammatory, redox reactions and signal transduction. It is available in supplemental form for gastrointes- tinal discomfort as an antiseptic. Today curcumin and turmeric still evokes great interest as a food additive, nutraceutical (or dietary supplement) and prospective candidate in medicine (Choudhary and Sekhon 2012, Goel at al. 2008, Gupta et al. 2013). Many health benefits, such as potential cancerchemotherapeutical properties, therapeutic benefits in several human oxidative stress models such as for Alzheimer's disease, ethanol induced oxidative injuries in brain, liver, heart, kidney and prevention of myocardial ischemic damage have been claimed for curcumin and these have generally been attributed to its radical- trapping antioxidant properties (Bargezar and MoosaviMohavedi 2011, Kumar et al. 2011). Curcumin is a highly pleiotropic molecule and preclinical study had formed a solid basis for evaluating curcumin's efficacy in clinical trials against a wide range of human diseases (Fig. 1). Hence, a new of interest, this time shifted to improvements in the active compounds of turmeric pharmacokintetics and delivery systems. Today, unlike many other natural compounds within the ethno-medicinal tradition, still evokes great interest as a food additive, nutraceutical (or dietary supplement) and 
prospective medicine (Gupta et al. 2013). Owing to its valuable properties almost 100 pharmaceutical and chemical companies are producing various curcumin products in the form of tablets, capsules, coloring agents, dairy, creams, drinks, extracts (from turmeric), gels, nasal sprays, and so on for daily and medicinal needs (Yallapu et al. 2012).

Turmeric is traditionally used a lot in the Middle East as a liver protector, a stimulant of bile duct, secretion, antiflatulent, diuretic, for curing catarrh, for the circulation, antifever, antiinflammatory and for rheumatisms or sprains (Kumar et al. 2011). Safety evaluation studies indicate that both curcumin and turmeric are well tolerated at a very high dose without any toxic effects.

Curcumin, a safe, affordable natural bioactive molecule of turmeric has shown efficacy against numerous human ailments and is one of the most extensively investigated natural compoud. However, its optimum pharmaceutical potential has been limited by its lack aqueous solubility and poor bioavailability due to poor absorption, rapid metabolism, and rapid systemic elimination. To mitigate the above limitations, various nanostructured water soluble delivery systems of curcumin and curcuminoids were recently developed to increase the solubility and bioavailability (Gounder and Lingamallu 2012, Anand et al. 2007, Yallapu et al. 2012, Gupta et al. 2013).

\section{Chemical composition of turmeric (Curcuma longa L.)}

Turmeric (Curcuma longa L.), a tropical herb of the Zingiberaceae family, native to southern Asia, primarily consumed in the form of powdered rhizoma, the yellow pigment is one of the major

\section{Cancer}

colorectal, pancreatic, breast, prostate, lung,head and neck cancer, multiple myeloma

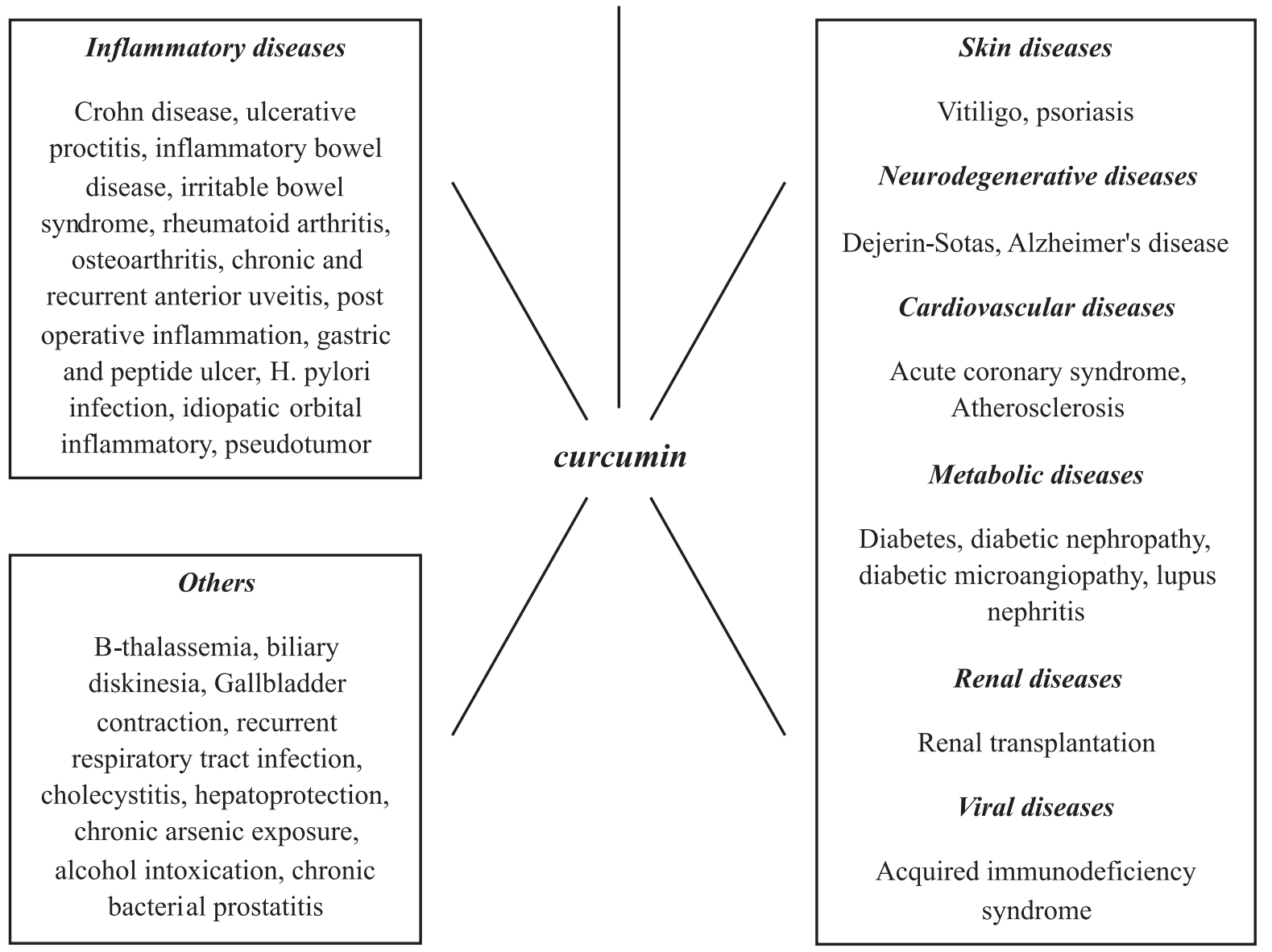

Fig. 1. Human diseases against which curcumin has exhibiting activity (Gupta et al. 2013). 
natural spice used for centuries in Indian cuisine. The curcuminoids and volatile oils, which are the major secondary metabolites of turmeric, have been shown to be largely responsible for the pharmacological activities of turmeric powder and extract from rhizomes. Its medicinal properties have been attributed mainly to the main compound of this group - curcumin - (1,7-bis(4-hydroxy3-methoxyphenyl)-1,6-heptadiene - 3,5-dione), often referred to as diferuloylmethane. Curcumin exists, predominantly as an aquilibrium of equivalent enol forms rather than tautomeric keto-enol mixture. Nevertheless the content of curcumin in turmeric is usually 4-5\%. Commercial curcumin (purity > $95 \%$ ) contains three major compounds: curcumin $(77 \%)$, demethoxycurcumin (17\%) and bisdemethoxycurcumin (3\%), together referred to as curcuminoids (Fig. 2, Gounder and Lingamallu 2012, Kumar et al. 2011).

Curcumin is considered hydrophobic, based on predicted values of $\log \mathrm{P}$ ranging from 2.56 to 3.29, which justifies such experimental observation at lipid membrane affinity, interaction with hydrophobic domains of proteins and ability to cross the blood brain barrier (Grynkiewicz and Slifirski 2012).

\section{Molecular pharmacology}

There is an extensive evidence indicating that curcumin interacts, directly or by modulating signaling pathways with many molecular target (Tab. 1, Gupta et al 2013), is a powerful antioxidant, which good electron transfer capability, which is considered to be ten times more effective in this respect than alpha-tocopherol, leads to secondary free radicals which considerably less harmful.

Curcumin also showed good electron-transfer capability, because of its unique structure and different functional groups, including a $\beta$-diketone several, electrons that have the capacity to conjugate between two phenyl rings (Fig. 3, Bargezar and MoosaviMovahedy 2011, Grynkiewicz and Slifirski 2012). Potent antioxidant due to its ability to scavenger the mutagenic/carcinogenic reactive oxygen species, additionally, data have provided interesting insingt into the immunomodulatory potential curcumin. This suggests that curcumin's reported favourable effect in arthritis, allergy, astma, atherosclerosis, heart disease, Alzheimer's disease, diabetes and cancer (Villegas et al. 2008).

It should not be ignored, its ability to up-regulate expression of some genes, in particular to enhance

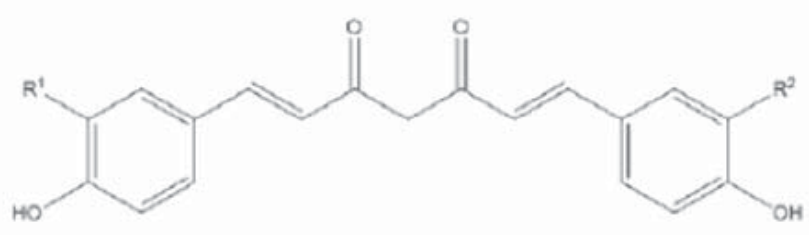

1 curcumin: $\mathrm{R}^{1}=\mathrm{R}^{2}=\mathrm{OCH}_{3}$

2 demetoxycurcumin: $\mathrm{R}^{1}=\mathrm{OCH}_{3} ; \mathrm{R}^{2}=\mathrm{H}$

3 bisdemetoxycurcumin: $\mathrm{R}^{1}=\mathrm{R}^{2}=\mathrm{H}$

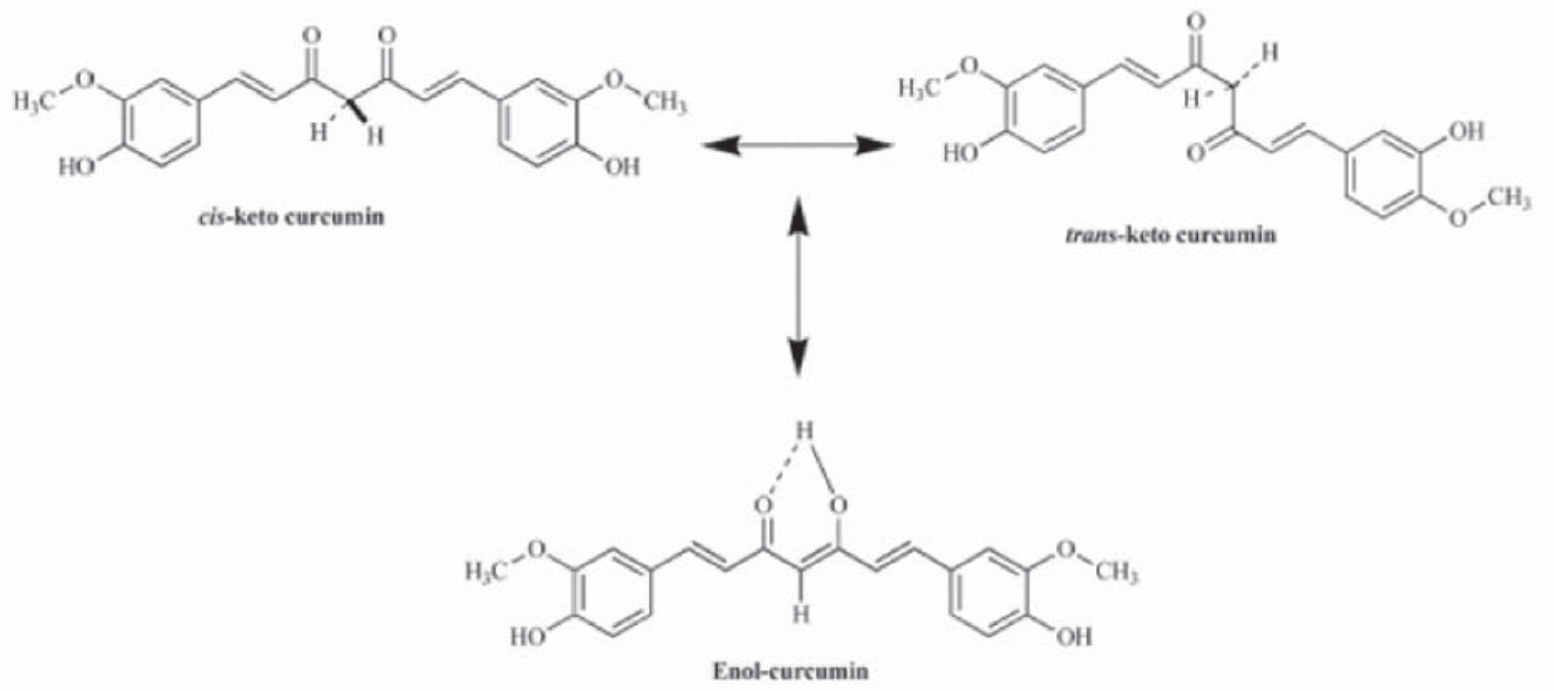

Fig. 2. Principal curcuminoids of turmeric shown as keto-enol equilibrium (Kumar et al. 2011). 


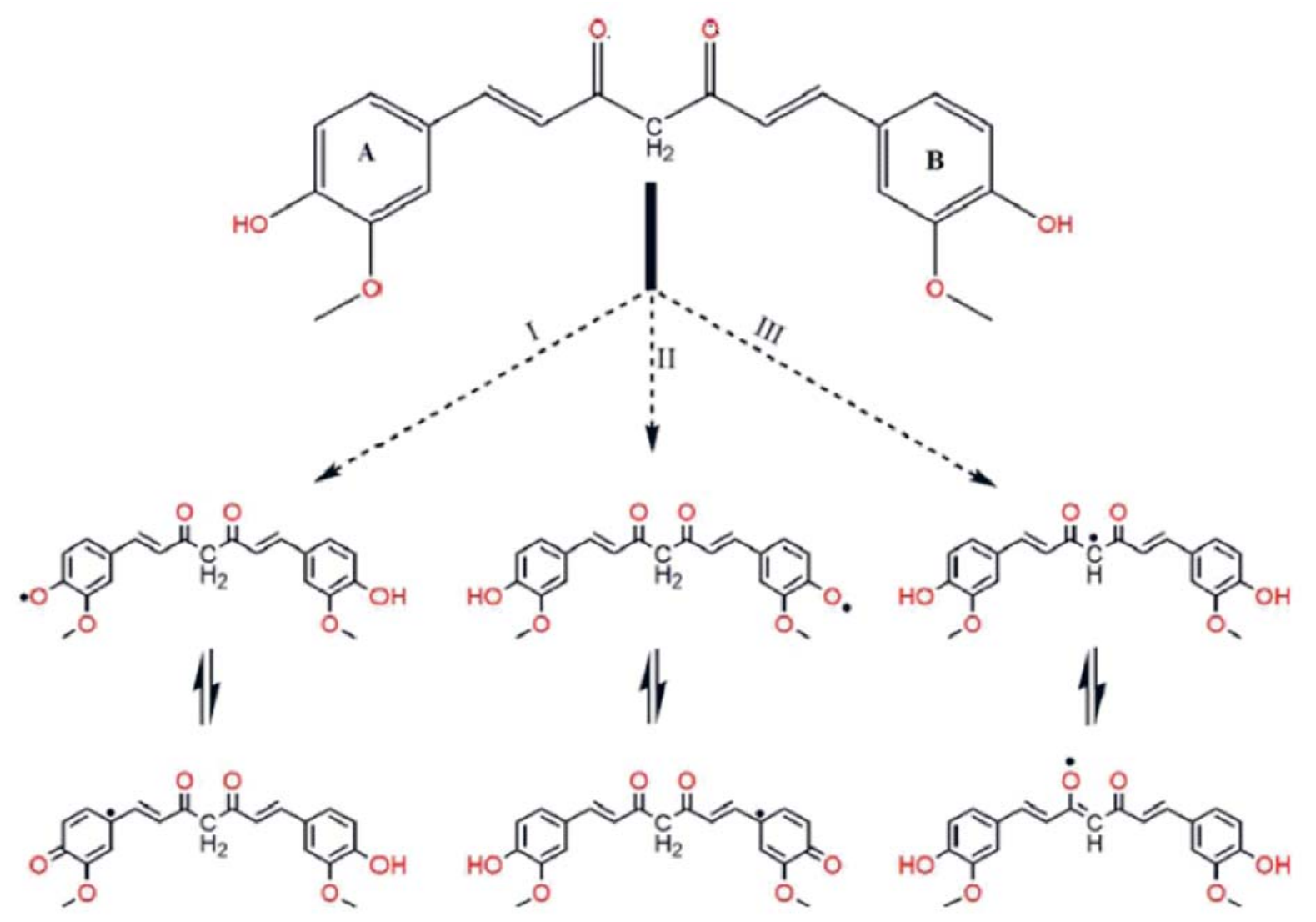

Fig. 3. The mechanism of probable three different sites of curcumin reactions with free radicals (Barzegar and Moosavi-Movahedi 2011).

production of inducible enzymes involved in biological redox processes (e.g. glutathione synthase GTS, cytochrome P 450 oxidases CYP 450, etc.).

There is no doubt that the most important biological actions of curcumin are those, connected with its highly selective interactions with certain proteins and leading to substantial inhibition of their activity. The antioxidant activity is connected with antiinflammatory effects supported by findings on inhibition of lipooxygenase and cyclooxygenase (LOX and COX, the key enzymes responsible for transformation of arachidonic acid to prostaglandins and leukotrienes) by natural and synthetic curcuminoids (Shezad et al. 2010). Inhibition of lipid peroxidation has been observed in many experimental models. Curcumin inhibits the production of proinflammatory cytokines (e.g. interleukines IL-1beta and IL-8), as well as tumor necrosis factor alpha (TNF-alpha), xanthine oxidase, inducible nitrogen oxide synthase (iNOS) (Grynkiewicz and Slifirski 2012, Aggarwal et al. 2007, Aggarwal and Sung 2008). The COX-2 and iNOS inhibiton are likely accomplished via curcumin's suppression of NF-kappaB, which is a ubiquitous transcription factor involved in the regulation of inflammation, cellular proliferation, transformation and tumorigenesis. Curcumin is thought to suppress NF-kappaB activation and pro-inflammatory gene expression by blocking phosphorylation of inhibitory factor 1-kappa B kinase (1 kappaB). Suppression of NF-kappaB activation subsequently down-regulates COX-2 and iNOS expression thereby inhibiting the inflammatory processes and tumorigenesis (Padhye et al. 2010, Prakobwong et al. 2011).

Additionally, a report on inhibition of thioredoxin reductase (TrxR, overexpressed in cancer cells), described irreversible modification of the enzyme by curcumin, as a results of a Michael addition reaction between the reductase rich in sulhydryl groups and the unsaturated conjugated ketone functionality of curcumin. The topic of covalent modification of proteins by curcumin warrants more research, like its interactions with lipids of cellular and organellar membranes which can lead to functional changes by modulating they fluidity and permeability. Two lines of research of the interaction of curcumin with proteins are purchased in inhibition studies (Grynkiewicz and Slifirski 2012, Gupta et al. 2013). For example, investigation of complexation of curcumin with soy lipooxygenase indicated formation of a metastable adduct with a new bicyclic derivate of curcumin with four newly formed chiral centers. This experiment indicates that initial complexes of 
Tab. 1. Molecular target of curcumin in human participants (Gupta et al. 2013).

\begin{tabular}{|c|c|}
\hline Disease & Biomarkers \\
\hline Colorectal cancer & GST $\downarrow, \mathrm{PGE}_{2} \downarrow \mathrm{M}_{1} \mathrm{G} \downarrow, \mathrm{TNF}-\alpha \downarrow, \mathrm{Bel}-2 \downarrow, \mathrm{p} 53 \uparrow, \mathrm{BAX} \uparrow$ \\
\hline Pancreatic cancer & MDA $\downarrow$, GSH $\uparrow$, IL- $6 \downarrow$, IL- $8 \downarrow$, IL-10 $\downarrow$, NF-kappaB $\downarrow$, COX-2 $\downarrow$, pSTAT3 $\downarrow$ \\
\hline Prostate cancer & PSA $\downarrow$ \\
\hline Multiple myeloma & Paraproteins $\downarrow$, NTT $\downarrow$, NF-kappaB $\downarrow$, COX-2 $\downarrow$, pSTAT3 $\downarrow$ \\
\hline Cancer lesions & Vitamin $\mathrm{C} \uparrow, \mathrm{E} \uparrow, \mathrm{MDA} \downarrow, 8-\mathrm{OHdG} \downarrow$ \\
\hline Head and neck cancer & IKK $\beta \downarrow$, IL- $8 \downarrow$ \\
\hline Inflammatory bowel disease & CRP $\downarrow, \mathrm{ESR} \downarrow, \mathrm{CDAI} \downarrow, \mathrm{p} 38 \mathrm{MAPK} \downarrow, \mathrm{IL}-1 \beta \downarrow, \mathrm{MMP}-3 \downarrow, \mathrm{IL}-10 \uparrow$ \\
\hline Osteoarthritis & CRP $\downarrow$, IL- $1 \beta \downarrow$, IL- $6 \downarrow$, sCD $40 L \downarrow$, sVCAM1 $\downarrow$, ESR $\downarrow$ \\
\hline Helicobacter pylori infection & sPGII $\downarrow$, sPGI $\downarrow$ \\
\hline Psoriasis & PhK $\downarrow, T R R \downarrow$, CD8+T cells $\downarrow$ \\
\hline Acute coronary syndrome & $\mathrm{TC} \downarrow, \mathrm{LDL} \downarrow, \mathrm{HDL} \uparrow, \mathrm{TG} \uparrow$ \\
\hline Atherosclerosis & Lipid peroxides $\downarrow$, TC $\downarrow$, HDL $\uparrow$ \\
\hline Type 2 diabetes & MDA $\downarrow$, ET- $1 \downarrow$, IL- $6 \downarrow$, TNF- $\alpha \downarrow$, HOMA- $\beta \uparrow$, adiponectin $\uparrow$, C-peptide $\downarrow$, HOMA-IR $\downarrow$ \\
\hline Diabetic nephropathy & TGF- $\beta \downarrow$, IL- $8 \downarrow$ \\
\hline Renal transplantation & Creatinin $\downarrow, \mathrm{HO}-1 \uparrow$ \\
\hline в-Thalassemia & MDA $\downarrow$, SOD $\downarrow$, GSH-Px $\downarrow$, NTBI $\downarrow$, GSH$\uparrow$ \\
\hline Hepatoprotection & AST $\downarrow$, ALT $\downarrow$, bilirubin $\downarrow$, ESR $\downarrow$ \\
\hline Arsenic exposure & Catalase $\uparrow, \mathrm{GSH} \uparrow, \mathrm{SOD} \uparrow, \mathrm{GPX} \uparrow, \mathrm{ROS} \downarrow$ \\
\hline
\end{tabular}

$\downarrow$ downregulation, $\uparrow$ upregulation, ALT alanine transaminase, AST aspartate transaminase, Bax Bel 2-associated X protein, Bel-2 B cell lymphoma-2, CDAI Crohn disease activity index, COX-2 cyclooxygenase 2, CRP C- reactive protein, GSH glutathion, GST glutathion S-transferase, GPX gluatthion peroxidase, HDL high density lipoprotein, LDL low density lipoprotein, HOMA homeostasis model assessment, IL interleukin, MMP-3 matrix metalloproteinase, NF-kappaB nuklear factor kappaB, $\mathrm{PGE}_{2}$ prostaglandin $\mathrm{E}_{2}$, pSTAT3 - phosphorylated form of signal transducer and activator of transcription 3, SOD - superoxide dismutase, ROS - reactive oxygene species, CD cluster of differentiation, ESR erythrocyte sedimentation rate, ET1 endothelin 1, IR insulin resistance, MAPK nitrogen activated protein kinase, MDA malondialdehyd, $\mathrm{M}_{1} \mathrm{G}$ pyrimido[1,2- $\alpha]$ purin-10(3H)-one, TNF- $\alpha$ tumor necrosis factor- $\alpha$, PSA prostate-specific antigen, NTT N-telopeptide of type 1 collagen, 8-OHdG 8-hydroxydeoxyguanosine, TC total cholesterol, TG triglyceride, PhK phosphorylase kinase, TRR transferrin receptor.

curcumin with hydrophobic pockers of some proteins can relatively easily undergo transition from hydrogen bonding controlled structure to a new covalent bonding.

Perhaps even more important are other target categories receptor, growth factors, transcription factors, inflammatory cytokines, cell adhesion molecules and gene expression regulators. The effect of activity modulation of the mentioned targets with curcumin can be arranged along know signaling pathways. For example, curcumin down regulates expression and activity of epidermal growth factor. Alternatively, in recent years many experiments have focused on nuclear factor kappa B (NF-kappaB) as an agent who is constitutively over-expressed from inflammation to cancer, controlling through various downstream effectors, such important stages as tumor growth, invasion, angiogenesis and metastasis. The collected pharmacological data demonstrated, that curcumin inhibits NF-kappaB activation, not by chemical modification of the protein, but rather by neutralizing its activators, or their products
(Prakobwong et al. 2011, Grynkiewicz and Slifirsky 2012, Basile et al. 2009).

As has been stressed, an overall effect of pleiotropic action of curcumin at the cellular level is that it acts as a chemosensitiser and radiosensitiser for tumors and as chemoprotector and radioprotector for normal tissues (Goel and Aggarwal 2010, Hoon Bong 2000).

Comprehensive reviews in the literature characterized curcumin as an excellent molecule among many other naturally occuring compounds for cancer therapeutics. Pleiotropic properties of curcumin molecule enable it to target the genome (DNA), messenger (RNA) and enzymes (proteins) within cells, actions that can be sequencial or simultaneous. Unlike other chemotherapeutic agents, curcumin exhibit pleiotropic properties that involve the modulation of nuclear factor - kappaB (NF-kappaB), transcription factor activator protein-1 (AP-1), tumor protein 53 (p53), mitogen-activated protein kinase (MAPK), nuclear beta-catenin signaling (Yallapu et al. 2012, Aggarwal et al. 2003). 
Epigenetic, heritable changes in gene expression occurring without a change in the DNA sequence, constitutive an important mechanism by which dietary compounds can selectively activate or inactivate gene expression. Curcumin, has recently been determined to induce epigenetic changes (changes in DNA methylation, histone modification, and alteration in microRNA (miRNA)) (Reuter at al. 2011), which may lead to increased sensitivity of cancer cells to conventional agents and thus inhibition of tumor growth. Curcumin is one of powerful and promising chemopreventive and anticancer agents with great potential as epigenetic agents. Unlike genetic changes, epigenetic changes can be modified by the environment, diet, or pharmacological intervention. The development of curcumin for clinical use as a regulator of epigenetic changes, however, needs investigation to determine novel and effective strategies either alone or in combination with other anticancer agents for improving cancer treatment. Indeed, some dietary polyphenols may exert their chemopreventive effects in part by modulating various compounds of the epigenetic machinery in human (Link et al. 2010, Padhye et al. 2010). Curcumin has been shown to be promising anticancer agent in several in vitro and animal studies.

\section{Target pathologies}

Curcumin, known thousands of years in Ayurvedic medicine and popular as a spice in Asian cuisine, relatively recently was considered a drug candidate in Western medicine. Multifactorial anti-inflammatory action of curcumin and curcuminoids, very low ulcerogenic index and a lack of antipyretic effects were underlined as arguments for potential application in treatment of rheumathoid arthritis, with some advantage over non steroidal anti-inflammatory drugs, as shown antirheumatic activity identical to that of phenylbutazone by improvement in joint swelling, morning stiffness, and walking time (18 pacients with rheumatoid arthritis at a dose that corresponded to $1.2 \mathrm{~g}$ of curcumin per day for 2 weeks) (Gupta et al. 2013).

Animal studies involving rats and mice, as well as in vitro studies utilizing human cell lines, have demonstrated curcumin's ability to inhibit carcinogenesis at three stages: tumor promotion, angiogenesis, and tumor growth. In two studies of colon and prostate cancer, curcumin inhibited cell proliferation and tumor growth (Kumar et al. 2011). Curcumin has been demonstrated to be an effective modulator of the activity of a majority of mediators responsible for tumor promotion and progression (Grynkiewicz and Slifirski 2012). Preclinical studies of curcumin have shown the ability to inhibit carcinogenesis in various types of cancer including breast, gastric, hepatic, leukaemia, oral epithelial, ovarian, pancreatic, prostate. As a results there is extensive interest in the clinical development of this compound as a cancer chemopreventive and/or chemotherapeutic agent as evidenced by the development of phase I clinical trials and current enrolment in phase II clinical trials (Villegas et al. 2008)

The structure and properties of curcumin can form the basis for development of antitumor compounds that more effective against both chemosensitive a multidrug-resistant cells.

The clinical trials conducted thus far have indicated the therapeutical potential of curcumin against a wide range of human diseases (Fig. 1). In these clinical trials, curcumin has been used alone or its combination with other agents such as quercetin, piperine, resveratrol, catechin, soy isoflavone (naturally compounds), or gemcitabine, $\mathrm{N}$-acetylcystein, mesalamine, prednisone, sulfasalazine, lactoferrin (Gupta et al. 2013).

\section{Attempts to manage bioavailability and pharmakokinetics}

For centuries it has been known that turmeric exhibits anti-inflammatory activity, but extensive research performed within the past two decades has shown that this activity of turmeric is due to curcumin (diferuloylmethane). It is not clear whether all the three natural analogues (curcumin, demethoxycurcumin and bisdemethoxycurcumin) exhibit aqual activity. Although in most systems curcumin was found to be most potent. There are also suggestion that the mixture of all three curcuminoids is more potent than either one alone (Balaji and Chempakan 2010).

Curcumin (diferuloylmethan) regulate numerous transcription factors, cytokines, protein kinases, adhesion molecules, redox status and enzymes that have been linked to inflammation. The process of inflammation has been shown to play a major role in most chronic illnesses, including neurodegenerative, cardiovascular, pulmonary, metabolic, autoimmune and neoplastic diseases (Aggarwal and Harikumar 2009). It has shown therapeutic potential against a number of human diseases, it's a prominent candidate for treating antiinflammatory, cystic fibrosis, Alzheimer's and malarial diseases in addition to cancer (Yallapu et al. 2012). Common to all of these studies have been the safety, tolerability, and non toxicity of this polyphenol, even at doses up to $8 \mathrm{~g}$ per day. The underlying mechanism for curcumin's clinical efficacy seems to be modulation numerous intracellular signaling molecules. However, because of the complex nature of the diseases, the underlying mechanism in many case remain unclear. 
The results from several pilot and phase I clinical trials in patients and volunteers confirm that low systemic bioavailability is achieved after oral dosing and it is related to rapid first pass metabolism and some degree of intestinal metabolism (curcuminconverting enzymes are of intestinal microbial origin - they result from partial or even total hydrogenation of the heptatrienone carbon chain). Products of metabolism are glucuronates and sulphates. A major limiting factor of curcumin is its low solubility in water (i.e. $0.4 \mu \mathrm{g} / \mathrm{ml}$ at $\mathrm{pH} 7.3$ and soluble curcumin molecule are extremely sensitive at physiological pH (Yallapu et al. 2012). Although 10 or $12 \mathrm{~g} / \mathrm{ml}$ of curcumin administered orally in human showed curcumin level in serum to be approximately $50 \mathrm{ng} / \mathrm{ml}$, this resulted in a minimum availability of curcumin in the blood circulation to achieve its therapeutic efects (Grinkiewicz and Slifirski 2012, Aggarwal and Harikumar 2009). Proven explorations suggest that improved curcumin properties, targeted delivery, tissue distribution and bioavailability in tumors can be efficiently achieved in the presence of an adjuvant, piperin, an inhibitor of glucuronidation, administered concominantly with curcumin to increase its biovavailability (Pathak and Khandelwal 2008). When $20 \mathrm{mg}$ of piperine was administered orally with $2 \mathrm{~g}$ of curcumin to volunteers, serum levels were significantly enhanced at 1 hour's time, increasing total bioaviability by 20-fold (Grynkiewicz and Slifirski 2012).

Furthermore, it is interesting to highlight that the coadministration of piperin (a compound of pepper), results in a substantial increase of curcumin bioavailability i both rats and human, compared to curcumin alone. Similarly, the association with quercetin or epigallocatechin gallate, compounds of gree tea, induced a synergistic effect, enhances antioxidative and hepatoprotective effect in paracetamol induced oxidative stress in animals, However, induced a synergistic effect on the growht of premalignant and malignant oral epithelial cells. Then, use of carefully chosen combination of discrete dietary agensts, formulated as "nutraceutical", might the beneficial to exploit the synergy among them (Villegas et al. 2008). Enhanced activity could be because of piperin and quercetin contribution to decrease curcumin metabolism by inhibiting enzymes and thus confirming the set hypothesis. The hepatoprotective action of combinatorial extract also provides a scientific basis for its usage in the traditional systems of medicine, for the managment of hepatotoxicity (Mehta et al. 2012). Turmeric (Curcuma longa L.) has enormous potential for a variety of diesases including hepatotoxicity. Therefore there is an extensive need for combinatorial extract which may enhance bioavailability of oral curcu- min by inhibiting the enzymes responsible for the metabolism of curcumin.

The present data of other experiment support potent lipid lovering action of combination consisting curcumin with piperine and quercetin $(94: 1: 5)$ in the high-fat diet induced obesity, glucose intolerance and oxidative stress induced rats as hypolipidemic and antihyperglycemic effect (Ginpreet and Meena 2012). Curcumin antioxidant activity and its possible synergistic effects with other phytocompounds were also studied in vivo. Curcumin and resveratrol (3,5,4'-trihydroxystilbene) together resulted in a greater synergistic antioxidant effect than an average of individual activities. This synergy was significantly higher than that of curcumin together with the flavonol quercetin. Fig. 4 shown structure of the mentioned phytocompounds examined in the heme-enhanced oxidation assay (Aftab and Vieira 2010). Resveratrol, a polyphenolic compound found in grape vines (Vitis vinifera) and a variety of other plants, is a specific inhibition of cytokine induced NF-kappaB activation, has much less antioxidant ac-

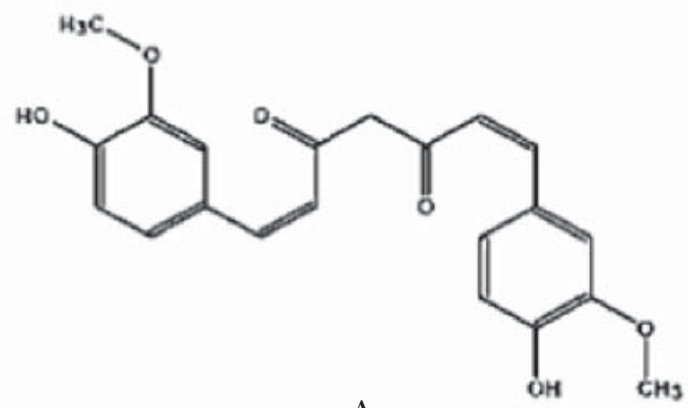

A<smiles>Oc1ccc(/C=C/c2cc(O)cc(O)c2)cc1</smiles><smiles></smiles>

Fig. 4. Structure of curcumin (A - keto form shown; enol form common), resveratrol (B) and quercetin $(\mathrm{C})$ examined in the heme-enhanced oxidation assay (Aftab and Vieira 2010). 
tivity in this assay but in combination with curcumin may have synergistic effects (Mobashedi et al. 2012). Complexes of curcumin with metal ions $\left(\mathrm{Zn}^{2+}, \mathrm{Cu}^{2+}\right.$, $\mathrm{Mg}^{2+}$ and $\mathrm{Se}^{2+}$ ) were found to be readily soluble in water-glycerol $(1: 1 \mathrm{w} / \mathrm{w})$ and quite stable towards light and heat. All complexes were found to provide a higher stability from curcumin alone (Zehib et al. 2010). Water soluble curcumin conjugates with two differently sized poly(ethylenglycol) molecules exhibit enhanced cytotoxicity as compared to curcumin alone and demonstrated some potential in anticancer treatment (Safavy et al. 2007). Various novel delivery systems have been proposed in recent year as means of improving the bioaviability of curcumin. They provide longer circulation, they increase the cellular permeability and they down metabolic transformation processes.

The key structural positions for the anticancer activities include the side aryl rings, conjugated double bounds, the central diketo function and modification of active methylene group. Among these, the substituents on active methylene group to hold the key for modulating the metabolic rates of curcumin. The difluorocurcumin condensate has shown metabolically stable allowing longer circulation time in biological systems and preferential accumulation in the pancreas in animal study and subsequently clinical trials for the use of these analogs for prevention of tumor progression and/or treatments of human malignancies (Padhye et al. 2010).

Animal studies also have shown that curcumin is rapidly metabolized, conjugated in the liver, and excreted in the feces, therefore having limited systemic bioavaiability. However, its optimum pharmaceutical potential as nutraceutical has been limited by its lack of aqueous solubility and poor bioavailability (low absorption, rapid metabolism and rapid system elimination). To mitigate to above limitations, recently various nanostructured watersoluble delivery systems were developed to increase the solubility and bioavailability of curcumin (Anand et al. 2007, Yallapu et al. 2012)

The present review summarizes the strategies using curcumin in various nanocarrier delivery systems to overcome poor solubility and inconsistent bioavailability and described the current status and challenges for the future. However, the future of such delivery technology will be highly dependent on the development of safe, non-toxic and non-imunogenic nanocarries (Gupta et al. 2012, Grynkievicz and Slifirski 2012, Yallapu et al. 2012, Chounhary and Sekhon 2012). Nanoparticle encapsulated curcumin increased bioavailability when compared with the conventional curcumin, protect from degradation, and increase its targeting capacity toward cancer and other diseases.
Various types of nanoparticles (NPs), such as polymer NPs, polymeric micelles, liposome/phospholipid, nano/microemulsion, nanogels, solid lipid NPs, polymer conjugates, self-assemblies and so one, are suitable for the delivery of an active form of curcumin not only to tumours (Yallapu et al. 2012, Grynkievicz and Slifirski 2012, Sou 2012).

\section{Drug delivery systems}

\section{Curcumin nanocrystal and conjugates}

Nanodrug crystals have a greater dissolution rate owing to a larger specific surface area. The aggregation of surfactant molecules, including sodium dodecyl sulphate, Tween 80, Triton $\mathrm{X}-100$ and pluronic polymers, form micelles at a critical micelle concentration and can provide the required influence to stabilize curcumin molecule. High- pressure homogenization ( $\mathrm{HPH}$ ) is a suitable method for reducing bulk curcumin into NPs. These formulations have a 10-14 fold greater absorption rate in Wistar strains rats given an oral treatment of $50 \mathrm{mg} / \mathrm{kg}$ of curcumin, compared with the same oral dose of free curcumin. The area under the plasma concentration was greater than for a curcumin solution.

Polymer-drug conjugates are considered to be alternative therapeutics from the nanoscale family. Two phenolic rings and active methylene groups are potential sites to conjugate any biomacromolecules onto curcumin. A recent patent technology based on a luteinizing hormone- releasing hormone (LHRH)curcumin conjugate has exhibit an improved antitumor effect in xenograft model of pancreatic cancer. Novel polyethylene glycosylated curcumin analogs, regulating the antioxidant defense system and act as modifiers for inflammatory diseases, are also used. PEGylated curcumin conjugates had a key role in growth inhibitory effects on a panel of human pancreatic cancer cell lines (Yallapu et al. 2012). However, the encapsulation of the curcumin in PLGA (poly lactic-co-glycolic acid) enhanced the bioaviability, had a longer half-life compared with the native curcumin and were found to destroy amyloid agregats, exhibit antioxidative property as well as. PLGA-curcumin nanoparticles can be used as a drug with multiple functions in treating Alzheimer's disease. Nanocurcumin enhance the clinical efficacy by enabling aqueous dispersion (Choudhary and Sekhon 2012).

Other researchers have synthesized polymeric nanoparticle encapsulated formulation of curcumin (nanocurcumin) utilizing the micellar aggregates of cross-linked and random copolymers of $\mathrm{N}$ isopropylacrylamide, with $\mathrm{N}$-vinyl-2-pyrrolidone and poly(ethyleneglycol)monoacrylate. Nanoparti- 
cles circumvent the pitfalls of the polyphenol poor solubility (Villegas et al. 2008).

\section{Curcumin emulsion, liposome and phytosome formulation}

Microemulsions are isotropic nanostructural, stable solutions comprising surfactant(s), oil and water. Curcumin-based microemulsions are expected to improve curcumin delivery via local and transdermal routes for skin cancer, psoriasis. Eucalyptolbased curcumin mikroemulsion have very high permeability and flux with moderate solubility on the cellular structure of skin.

Extensive research is currently focused on curcumin as a potential novel drug for near future to control various diseases including inflammatory disorders, carcinogenesis, Alzheimer's disease and oxidative stress-induced pathogenesis. Therapeutic utility of curcumin is limited, therefore methods are being developed through synthetic approaches to alter its properties or incorporate it into different host system to improve the curcumin delivery system. Phytosomes are novel complexes produced by a patented process whereby standardized turmeric extract or curcumin, are bound to phospholipid, mainly phosphatidylcholine, producing a lipidcompatible molecular complex (obtained by reaction of steichiometric amounts of phospholipid and the substrate in an appropriate solvent) known as phytosome technology. Phytosome exhibit a better pharmacokinetic and pharmacodynamic profile than conventional turmeric (Curcuma longa) extract or curcumin due to its enhanced capacity to cross the lipid-rich biomembranes and reach circulation, and has effectively enhanced the bioavailability of many popular herbal extracts, include milk thistle, Ginkgo biloba, grape seed, green tea, hawthorn, ginseng etc and can be further developed for clinical applicability of polyphenols and other poorly absorbed active compounds and extracts as nutraceuticals (Tab. 2, Maiti et al. 2007, Gupta and Dixit 2010). The results of ex vivo study show that phytosome complex has significantly increased absorption compared to curcumin, improved pharmacokinetics, and increased hepatoprotective and antioxidant activity. Phosphatidylcholine (from soybean, Glycine max), the major molecular building block of cell membranes is a compound miscible in both water and in lipid environments. In humans and other higher animals the phospholipids are also employed as natural digestive aids, the major molecular building block of cell membranes (Awasthi et al. 2011, Choubey 2011). Phospholipids have a dual solubility and act as an emulsifier. Phytosomes show a transition from hydrophilic environment into the lipophilic environment of the enterocyte cell membrane followed by an entry into cell finally reaching into blood (Amin and Bhat 2012).

Phosphatidylcholine is a bifunctional compound, the phosphatidyl moiety being lipophilic and the choline moiety being hydrophilic in nature. Molecules are anchored through chemical bonds to the polar choline head of the phospholipid and active compound or plant extract are protected from destruction by gastric secretion and gut bacteria owing to the gastroprotective property of phosphatidylcholine (Jain et al. 2010). Phytosome

Tab. 2. Some marketed phytosomes preparation and indication.

\begin{tabular}{|c|c|c|}
\hline Phytosomes & Phytoconstituents complexed & Indication \\
\hline Centella phytosome & Terpenes & Vein and skin disorders \\
\hline Echinacea phytosome & Echinacosides from Echinacea angustifolia & Nutraceutical, immunomodulator \\
\hline Ginkgo phytosome & $\begin{array}{c}24 \% \text { ginkgoflavonoids from } \\
\text { Ginkgo biloba }\end{array}$ & $\begin{array}{c}\text { Protects brain and vascular linings, } \\
\text { antiskin ageing }\end{array}$ \\
\hline Ginseng phytosome & $37.5 \%$ ginsenosides from Panax ginseng & Immunomodulator, nutraceutical \\
\hline Green tea phytosome & $\begin{array}{l}\text { Epigallocatechin 3-O-gallate from } \\
\text { Camelia sinensis }\end{array}$ & $\begin{array}{l}\text { Food product, systemic antioxidant, } \\
\text { cancer protectant }\end{array}$ \\
\hline Grape seed phytosome & Procyanidins from Vitis vinifera & $\begin{array}{l}\text { Nutraceutical, cardio-protective, } \\
\text { systemic antioxidant }\end{array}$ \\
\hline Hawthorn phytosome & Flavonoids from Crataegus sp. & Nutraceutical, cardio-protective, antihypertensive \\
\hline Olive oil phytosome & Polyphenols from Olea europaea oil & $\begin{array}{l}\text { Antioxidant, anti-inflammatory, } \\
\text { anti-hyperlipidemic }\end{array}$ \\
\hline $\begin{array}{l}\text { Panax ginseng } \\
\text { phytosome }\end{array}$ & $\begin{array}{l}37.5 \% \text { ginsenosides from roots of } \\
\text { Panax ginseng }\end{array}$ & Food product \\
\hline Super milk thostle extract & Silybin from Silymarin & Food product, antioxidant for liver and skin \\
\hline Silybin phytosome & Silybin from Silybium marianum & Hepatoprotective, antioxidant for liver and skin \\
\hline Silybin phytosome & Silybin from Silymarin & Antioxidant for liver and skin \\
\hline
\end{tabular}


structures contain the active compounds of the herb surrounded by the phospholipid. It has been shown that the main phospholipid substrate interaction is due to the formation of hydrogen bonds between the polar head of phsopholipid (i.e. phosphate and ammonium groups) and the polar functionalities of the substrate (the phenolic hydroxyls of curcumine). Several excellent phytocompouds and plant extracts have been successfully delivered in this way exhibiting remarkable therapeutic efficacy in animal as well as in human models as phytosomes. When treated with water, phytosomes assumes a micellar shape forming liposomal-like structure.

Likewise phytosomes, a liposome is formed by mixing water soluble compounds with phosphatidylcholine in definite ratio. Here, no chemical bond is formed; the phosphatidylcholine molecules surround the water soluble compounds (Fig 4). There may be hundreds or even thousand phosphatidylcholine molecules surrounding the water soluble compound, molecular complex depending on the compounds complexes, involving chemical bonds. This difference results in phytosome being much better absorbed than liposomes showing better bioavailability. In liposomes the active principle is dissolved in the internal pocket or it is floating in the layer membrane, while in phytosomes the active principle is anchored to the polar head to phospholipid.

In this way after screening of potential extract or active compounds from turmeric and phytosome can be developed for different therapeutic purposes like cardiovascular, antiinflammatory, immunomodula- tory, anticancer, antidiabetic etc or for prophylactic and health purposes as nutraceuticals (Choundhary and Sekhon 2012, Yallapu et al. 2012).

\section{Conclussions}

Regarding toxicity, as curcumin and curcuminoids from turmeric (Curcuma longa L.) are compounds of the diet there are nontoxic in nature. The pharmacological properties and application of curcumin are rapidly progressing. Clinical study suggested that curcumin exhibit diverse and potent array of pharmacological effects in almost all of the major organ systems of the human body. These include: anti-diabetic, anti-inflammatory, anticancer, antiaging, antioxidant, antibacterial activities, hepatorpotective, cardiovascular and neurodegenerative effects. Safety evaluation studies indicate that curcumin is well tolerated, at a very high dose without producing any toxic effect (even at doses up to $8 \mathrm{~g}$ ). The underlying mechanism for curcumin's clinical efficacy seems to be modulation of numerous signalling molecules the underlying mechanism in many cases remains unclear. Poor bioavailability and limited adverse effects reported by some investigators are a major limitation to the therapeutic utility of curcumin. Nanoparticle encapsulated curcumin increased bioavailability when compared with conventional curcumin and therapeutic efficacy in treatment various disorders. Only a few novel multifunctional and composite nanosystem strategies offer simultaneous therapy as well as imaging characteristics. Numerous preclinical studies have

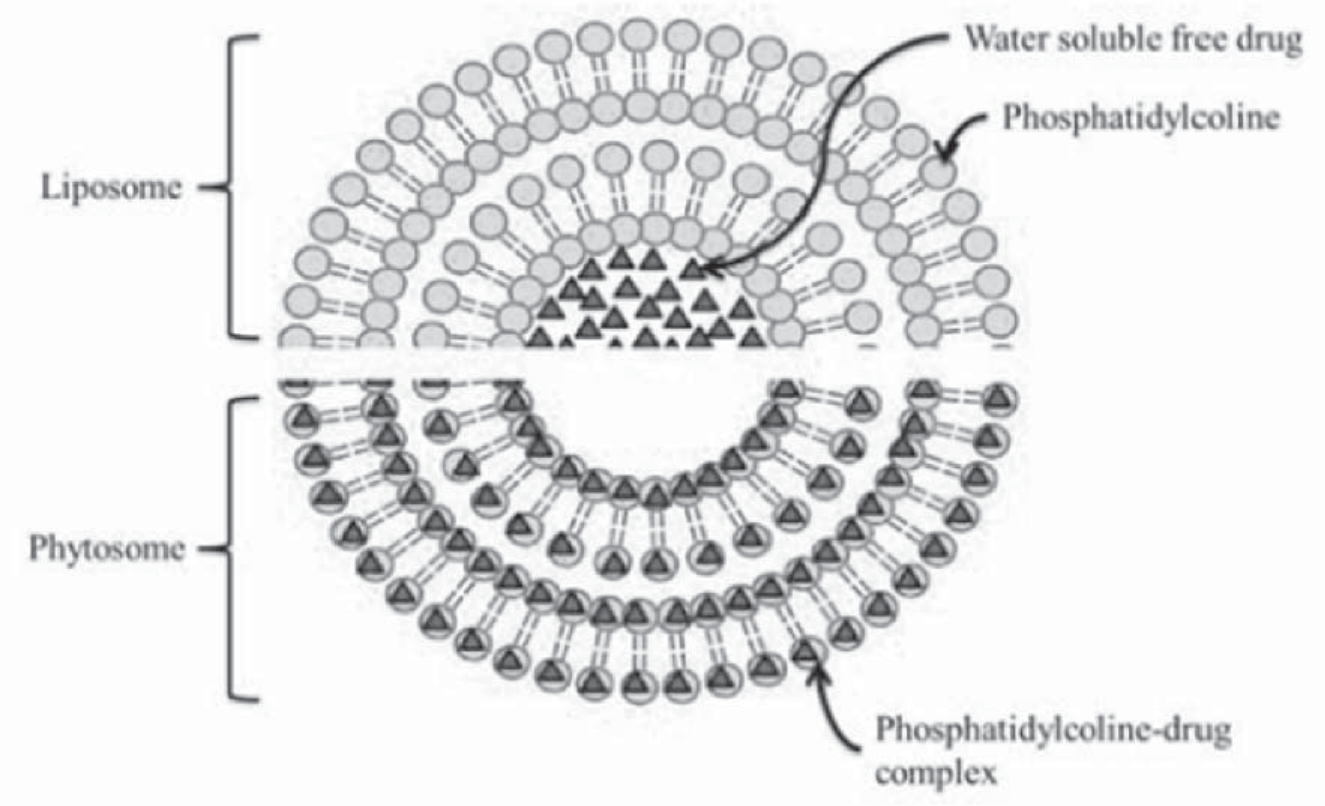

Fig. 5. Difference between phytosome and liposome. The molecular organisation of phytosomes (lower segment), liposome (upper segment) (Awasti et al. 2011). 
provided a solid basis for examining curcumin's efficacy against human diseases. We hope that the results from ongoing clinical trials will provide a deeper understanding of curcumin's therapeutic potential and will help to place this fascinating molecule at the front of novel therapeutics.

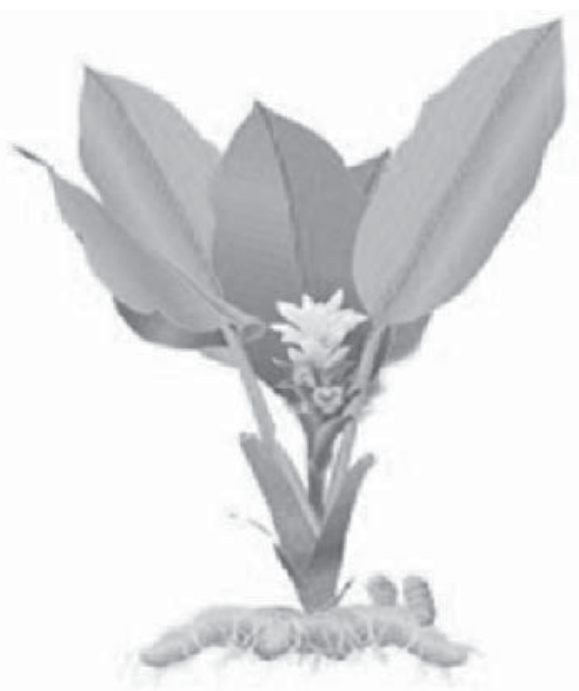

Fig. 6. Plant of Cucuma longa L. (turmeric).

\section{Acknowledgments}

This work was supported by The Agency of the Ministry of Education, Science, Research and Sport of the Slovak Republic for the Structural Funds of EU, OP RED of $E R D F$ in the frame of the Project "Evaluation of natural substances and their selection for prevention and treatment of lifestyle diseases" (ITMS 26240220040).

\section{References}

Aftab N, Vieira A (2010) Phytother Res 24: 500-502.

Aggarwal BB, Harikumar KB (2009) Int J Biochem Cell Biol 41: 40-59.

Aggarwal BB, Kumar A, Bhortii AC (2003) Anticancer Res 23: 363-398.

Aggarwal BB, Sundaram Ch, Malani N, Tchikawa H (2007) Adv Exp Med Biol 595: 1-75.

Aggarwal BB, Sung B (2008) Trends Pharmacol Sci 30: 85-94.

Amin T, Bhat SV (2012) Int J Adv Res Technol 1: 1-16.

Anand B, Kunnumakkara AB, Newman RA, Aggarwal BB (2007) Mol Pharm 4: 807-818.

Awasthi R, Kulkarni GT, Pawar VK (2011) Int J Pharm Pharm Sci 3: 1-3.

Balagi S, Chempakam B (2010) Food Chem Toxicol 48: 2951-2959.
Barzegar A, Moosavi-Movahedi AA (2011) Plos One 6: $1-7$.

Basile V, Ferrari F, Lazzari S, Belluri S, Pigmedoli F, Imbriano C (2009) Biochem Pharmacol 78: 13051315.

Berginc K, Trontelj J, Basnet NS, Kristl A (2011) Pharmazie 67: 518-524.

Ginpreet K, Meena C (2012) ISRN Pharmacology 1-7.

Goel A, Aggarwal BB (2010) Nutr Cancer 62: 919-930.

Goel A, Kunnumakkara AB, Aggarwal BB (2008) Biochem Pharmacol 75: 787-809.

Gounder DK, Lingmallu J (2012) Ind Crop Prod 38: 124-131.

Grynkiewicz G, Slifirski P (2012) Acta Biochim Pol 59: 4-11.

Gupta NK, Dixit VK (2010) J Pharm Sci 100: 19871995.

Gupta SC, Patchva S, Aggarwal BB (2013) AAPS J 15:195-218.

Hoom Bong P (2000) Bull Korean Chem Soc 21: 81-86.

Choubey A (2011) IJPSR 2: 807-815.

Choudhary N, Sekhon BS (2012) J Pharm Educ Res 2: 64-71.

Choudhary N, Sekhon BS (2012) J Pharm Res 3: 64-71.

Jain N, Gupta BP, Thakur N, Jain R, Banweer J, Jain DK, Jain S (2010) Int J Pharm Sci Drug Res 2: 224-228.

Kumar A, Dora J, Singh A (2011) Int J Aplied Biol Pharm Technol 2: 371-379.

Link A, Balaguer F, Goel A (2010) Biochem Pharmacol 80: $1771-1792$.

Maiti K, Mukherjee K, Gautait A, Saha BP, Mukherjee PK (2007) Int J Pharm 330: 155-163.

Mobasheri A, Henrotin Y, Bieselski HK, Shakibaci M (2012) Int J Mol Sci 13: 4202-4232.

Mehta A, Kaur G, Chintamaneni M (2012) Intern J Pharmacol 8: 101-107.

Padhye S, Chavan D, Pandey S, Deshpande J, Swamy KV, Sarkar FM (2010) Mini Rev Med Chem 10: 372-387.

Pathak N, Khandelwal S (2008) Breast Cancer Res Treat 122: 777-785.

Prakobwong S, Gupta SC, Kim JH, Sung B, Pinlaor P, Hiraku Y, Aggarwal BB (2011) Carcinogenesis 32: 1372-80.

Reuter S, Gupta SC, Park B, Goel A, Aggarwal BB (2011) Genes Nutr 6: 93-108.

Safavy A, Raisch KP, Mantena S, Sanford LL, Sham SW (2007) J Med Chem 50: 6284-6288.

Shezad A, Wahid F, Lee YS (2010) Arch Pharm 343: 489-499.

Sou K (2012) Recent Patents Nanomedicine 2: 133-163.

Villegas J, Sánchez-Fidalgo S, Alrcon de la Lastra C (2008) Mol Nutr Food Res 52: 1040-1061.

Yallapu MM, Jaggi M, Chanhan SC (2012) Drug Discovery Today 17: 71-80.

Zebib B, Mouloungui Z, Noirot V (2010) Bioinorganic Chem Appl 2010: 1-8. 